\title{
Molecular Imaging in Therapeutic Efficacy Assessment of Targeted Therapy for Nonsmall Cell Lung Cancer
}

\author{
Yanni Hu, 1, 2, 3, 4 Mei Tian, 1, 2, 3,4 and Hong Zhang ${ }^{1,2,3,4}$ \\ ${ }^{1}$ Department of Nuclear Medicine, Second Affiliated Hospital of Zhejiang University School of Medicine, Hangzhou 310009, \\ Zhejiang, China \\ ${ }^{2}$ Medical PET Center, Zhejiang University, Hangzhou 310009, China \\ ${ }^{3}$ Institute of Nuclear Medicine and Molecular Imaging, Zhejiang University, Hangzhou 310009, China \\ ${ }^{4}$ Key Laboratory of Medical Molecular Imaging of Zhejiang Province, Hangzhou 310009, China
}

Correspondence should be addressed to Hong Zhang, hzhang21@gmail.com

Received 28 December 2011; Accepted 30 January 2012

Academic Editor: Yasuhisa Fujibayashi

Copyright ( 2012 Yanni Hu et al. This is an open access article distributed under the Creative Commons Attribution License, which permits unrestricted use, distribution, and reproduction in any medium, provided the original work is properly cited.

\begin{abstract}
Membrane distillation is a thermally driven membrane process for seawater desalination and purification at moderate temperatures and pressures. A hydrophobic micro-porous membrane is used in this process, which separates hot and cold water, allowing water vapor to pass through; while restricting the movement of liquid water, due to its hydrophobic nature. This paper provides an experimental investigation of heat and mass transfer in tubular membrane module for water desalination. Different operating parameters have been examined to determine the mass transport mechanism of water vapor. Based on the experimental results, the effects of operating parameters on permeate flux and the heat transfer analysis have been presented and discussed in details.
\end{abstract}

\section{Introduction}

Lung cancer is one of the most common cancers in the world, as well as the leading cause of cancer death in men and the second leading cause in females globally [1]. Nonsmall cell lung cancer (NSCLC) accounts for more than $85 \%$ of all lung cancers [2]. The prevailing treatment options for NSCLC comprise surgery, radiotherapy, and chemotherapy, used either alone or in combination. Although local treatment modalities like surgery and radiotherapy can provide the chance for cure in early stages of NSCLC, about 54\% NSCLC patients present with a metastasis disease at diagnosis, with an overall 5-year relative survival $3.8 \%$, as estimated in 17 SEER geographic areas in America during 20012007 [3]. However, the option for these patients with advanced NSCLC whose lesions are mostly unresectable is limited to systemic therapy, where chemotherapy plays a predominant role. The use of chemotherapy in the treatment of this frustrating malignancy, which had a median survival measured in weeks or months in early days [4], was once a controversial issue concerning reproducible toxicity and questionable activity [4-6]. However, with validations of increasing publications of meta-analyses and randomized trials, especially with the advent of novel cytotoxic drugs with less toxicity and more activity, chemotherapy has become the mainstream of the treatment for advanced NSCLC. As an updated meta-analysis of data from 2714 patients of 16 randomized controlled trials revealed, compared with supportive care alone, chemotherapy with supportive care improves 1-year survival rate from $20 \%$ to $29 \%$ in all patients with advanced NSCLC [7]. Chemotherapy including platinum agents and the third generation drugs produces a cytotoxic effect by blocking cell division or DNA replication. Lots of randomized clinical studies in an effort to improve survival and life quality focused on the efficacy of differing combinations of chemotherapeutic drugs and revealed that variant combinations of chemotherapy agents produce similar response rate and survival $[8,9]$. It is generally accepted that the efficacy of chemotherapy for advanced NSCLC has reached a plateau [10], with a response rate of $25-35 \%$, time to progression (TTP, the time from randomization until objective tumor progression) 4-6 months, a 1-year survival 
rate of 30-40\%, and a median survival of 8-10 months [11]. Overall, the prognosis for advanced NSCLC remains poor.

With increasingly researches on molecular pathways of NSCLC in the last decade, aberrations in signaling pathways and molecules of tumor cells which promote tumor survival, proliferation, metastasis, and neovascularization have come to light. The advent of targeted therapy which acts selectively on the tumor-specific molecular pathways, and biomarkers detection which indicates a likely response to a specific therapy and guides the treatment choice [12], has brought the treatment strategy for NSCLC from empiricism into a new era of personalized therapy. The management of tumor is not "trial and error" modality any longer, but more predictive and efficient, with enhanced sensitivity to therapy and reduced unnecessary toxic effect and costs of likely ineffective treatment [13]. Targeted agents, composing mostly small molecule inhibitors and monoclonals antibodies, block signaling pathways by binding to intracellular domain to inhibit downstream signaling or to extracellular domain of surface receptor and activating immune mechanisms [14]. The target-signaling pathways or molecules such as epidermal growth factor receptor (EGFR) and vascular endothelial growth factor receptor (VEGFR) have already yielded significant improvements in response rate and progression-free survival (PFS, the time from randomization until objective tumor progression or death) used alone or in combination with chemotherapy compared with standard chemotherapy alone in large randomized clinical studies [15-19], and drugs of these categories such as erlotinib and bevacizumab have obtained FDA approval and have been recommended by National Comprehensive Cancer Center Network (NCCN) guidelines for subgroups of advanced and metastatic NSCLC. Many other targeted drugs acting on various pathways, for instance, heat shock protein (HSP)90 inhibitors, insulin growth factor-1 receptor (IGF-1R) inhibitor, poly(ADP-ribose) polymerase (PARP) inhibitors, mammalian target of rapamycin (mTOR) inhibitors, histone deacetylase inhibitors and anaplastic lymphoma kinase (ALK) inhibitors, have also shown promising prospect in clinical trials [20]. Recent reviews have been published of development on these targeted therapies for NSCLC $[21,22]$.

Despite the ever-increasing novel drugs and differing combinations of standard drugs for the improvement of survival of the patients with advanced NSCLC, a significant proportion of patients exhibit or acquire resistance and eventually experience progression [23]. Even in patients with a positive predictive biomarker undergoing a targeted therapy that is likely to be effective, primary resistance is shown in about $30 \%$ overall, and disease recurrence occurs uniformly in patients with an initial response [2427]. Moreover, although EGFR mutations in tumors are believed to be an important predictor of response to EGFR inhibitors, studies demonstrate that patients who benefit from EGFR inhibitors are not limited to those whose tumors have EGFR mutations [28]. Therefore, valid strategy for response prediction to help clinical management is still required.

Furthermore, after disease progression, patients often get to be unsuitable to receive further treatment due to declining performance status and increased symptom burden [2932]. Therefore, it is of great importance to identify early whether a patient is likely to respond to the current therapy and whether a resistance has occurs, for it enables an early termination of ineffective therapy and avoidance of unnecessary toxic effect or costs for those nonresponders, as well as more chance to receive a more likely to be effective therapy. In addition, as more novel drugs come into clinical trials with inconclusive efficacy, an early and valid identification of therapeutic efficacy helps to shorten the time for assessment and reduce cost.

While improved survival has been widely accepted as the "gold standard" for establishing clinical benefit in clinical trials, as an ultimate and long-term outcome, survival is not practical for early identification of therapy effect. As a consequence, valid surrogates for shortening the time for therapeutic efficacy assessment have been applied in clinical trials, which can reflect change of tumor and help to make prediction of long-term outcomes. Like tumor markers such as CA-125 in ovarian cancer has been already used as standard markers in the objective response assessment [33], in the context of lung cancer today, imaging that can provide an insight of tumor condition has long been applied in measurement of antitumor effect. The imagingbased endpoints like objective response, PFS, and TTP are commonly used in stage II and III clinical trials. Given the importance of early assessment of therapeutic efficacy in clinical practices and investigational therapies, appropriate imaging modalities as an essential part in response assessment are essential. In this review, we will discuss the current imaging modalities, especially molecular imaging, that applied in efficacy evaluation of targeted therapy for advanced NSCLC.

\section{Current Imaging Methods in Efficacy Assessment of Treatment for NSCLC}

2.1. Computed Tomography (CT). With the rapid development of cytotoxic drugs since 1960s, there has been an intense need for an acknowledged standard in anti-tumor effect assessment. Since the World Health Organization (WHO) first introduced response criteria of cancer in 1979 [34] in an effort to introduce a common language to report results of investigational antitumor therapies, anatomical imaging has become the standard way to determine oncology response in solid tumors. Because of some limitations of the WHO criteria, an international working group published the Response Evaluation Criteria in Solid Tumors (RECIST) guideline in 2000, and updated it in 2009. These criteria have determined tumor response as complete response, partial response, progression disease, and stable disease, according to the change of target lesions size, and have become the main standards in clinical trials of anti-tumor therapies for NSCLC where the primary endpoints are objective response or progression.

Tumor response is believed to be an indicator of drug effect in traditional anti-tumor trials and was approved by the FDA in 1996 to be used in investigational oncological 
treatments, as to accelerate approval of new anti-tumor drugs [35], for a reason that tumor is not likely to shrinkage spontaneously. Lots of trials demonstrated that objective tumor response is associated with longer survival in chemotherapy for solid tumors [36-39], yet whether tumor response can predict therapy efficacy remains an issue open to disputes [40-42]. At the same time, other endpoints such as disease control rate (consisting of CR, PR, and SD), PFS, or TTP [43] are increasingly proved to be more valid measures for therapy efficacy assessment in clinical trials [44-46]. These anatomybased endpoints, specified by those criteria, are commonly used in investigational trials of advanced NSCLC. CT with its high resolution, moderate costs, and availability has been historically the mainstay in measurement of lung cancer sizes and still the standard method in present. In addition, it is also the recommended method in clinic for surveillance and instructs clinical management.

However, since targeted therapy came into evaluation, there are concerns about whether morphology-based criteria that was established in cytotoxic therapy trials is an appropriate metrics in efficacy assessment of targeted-therapy, where tumor shrinkage is not necessary due to an effect of delaying the tumor growth. It has been observed in diverse tumors types like metastatic renal cell cancer and GIST [47, 48], as well as NSCLC [49-51], that relationship between tumor regression and survival varied widely. Because stability of disease cannot be interpreted as ineffectiveness of a targeted therapy, the response defined in change of dimension as a measure of efficacy in targeted therapy is inappropriate. In this case, endpoints such as PFS or TTP, which focus on progression rather than regression, might be more appropriate and are commonly utilized in clinical studies of targeted therapy. However, these metrics associated with progression need regular evaluation in trials and cannot provide information about treatment efficacy before tumor enlargement occurs and hence cannot be an early predictor of treatment efficacy, which is vital to optimizing a patient's management in clinic or improving efficiency of clinical trials.

2.2. Dynamic Contrast-Enhanced CT (DCE CT). Regarding the limitation of size-based methodology, metrics other than size is also proposed to provide information about tumor response to targeted therapy. DCE CT is also known as functional $\mathrm{CT}$, which can provide information about perfusion. Tumor perfusion versus whole-body perfusion can be measured on images generated following an intravenous injection of a contrast agent. A reduction in tumor perfusion is demonstrated in NSCLC patients responding to chemotherapy [52]. Similar result is also yielded in a study on targeted therapy that has an effect of antiangiogenesis for NXCLC in monitoring response of NSCLC to [53]. In this study, tumor perfusion is measured with DCE CT at week 3 and week 6 after starting treatment, and a reduction of tumor blood flow is observed at week 6. Meanwhile, tumor density used in combination with size has become a new imaging paradigm in assessment of targeted therapy for GIST [54] and metastatic renal cell carcinoma [55]. However, since there is no such study or criteria in NSCLC, whether combination of tumor density and size is a feasible metrics in evaluation of therapy for NSCLC is still unclear.

2.3. Dynamic Contrast-Enhanced Magnetic Resonance Imaging (DCE MRI). Although MRI used limitedly in imaging of NSCLC, DCE MRI has been used in evaluation of NSCLC perfusion. With this modality, quantitative parameters reflecting tumor microvessel perfusion, permeability can be obtained [56]. DCE MRI has been verified as a potential role in efficacy assessment of both cytotoxic agents and antiangiogenesis targeted-therapy for NSCLC $[57,58]$.

2.4. Magnetic Resonance Spectroscopy (MRS). By utilizing the special property of nuclear isotopes in a strong magnetic field, MRS gives information of tissue metabolites [59]. As a molecular imaging modality, MRS has a bright prospect in personalized medicine. It has been reported that the accuracy in discrimination between normal lung and each cancer type with proton MRS (1H MRS) was up to 81.5 to $90.7 \%$ [60]. However, there is no utilization of MRS available in response assessment area in NSCLC up to date.

2.5. Positron Emission Tomography (PET). The advent of PET brings about a totally new era of molecular imaging. By detecting the distribution of the radionuclide-labeled tracer that is involved with some specific physiological process within the body, PET makes metabolic change possible to be visualized in vivo, which is fundamentally different from conventional anatomy-based imaging modalities. Because biological features of tumor tissues are usually different from that of normal tissues, this functional imaging technology that makes qualitative, quantitative, and semiquantitative analysis available is especially useful in oncology imaging, playing a role in disease diagnosis, staging and restaging, and therapeutic response evaluation. In addition, since there are various biologic compounds participating different physiological processes, by developing diverse radionuclide-labeled tracers, we can acquire tumor information in an optimal way according to specific tumor or explore different physiologic features of tumor. While depicting an imaging on molecular characteristics of different tissues, PET can sometimes be imprecise in anatomical location. For this reason fusion of PET with traditional modality is developed and comes into application, combining molecular formations with highresolution anatomical structures. The multimodalities of PET/CT and PET/MRI are currently in practical application with promising prospect.

The positron-emitting isotopes of oxygen, carbon, nitrogen, and fluorine which are the building components of biological substances are the commonly used labels for tracers for PET. A large spectrum of radiopharmaceuticals has been developed associating with various metabolic pathways. 2$\left[{ }^{18} \mathrm{~F}\right]$ fluoro-2-deoxy-D-glucose $\left({ }^{18} \mathrm{~F}-\mathrm{FDG}\right)$ reflecting glucose metabolism is most widely used among all the tracers, with general acceptance in assessment of oncologic therapies. ${ }^{18} \mathrm{~F}$ FDG is a modified glucose molecular where the hydroxylgroup $(\mathrm{OH})$ of glucose is replaced with ${ }^{18} \mathrm{~F}$, which has 
a half-life of 110 minutes. Once being taken up into a cell, it cannot be further metabolized. Therefore, different levels of accumulation of ${ }^{18} \mathrm{~F}-\mathrm{FDG}$ are reflections of diverse energetic need. It is rational to consider that malignant cells will decrease glucose requirement due to direct or indirect damages. Clinical investigations have validated a reduction in ${ }^{18}$ F-FDG uptake that occurs in oncological tissues after anticancer treatment in various tumors including NSCLC and is closely correlated with final outcome of therapy [61]. 3 - $\left[{ }^{18} \mathrm{~F}\right]$ fluoro-3-deoxy-L-thymidine $\left({ }^{18} \mathrm{~F}\right.$-FLT $)$, a thymidine analogue, as a biomarker of proliferation, is another tracer often investigated in studies on NSCLC.

Radioimmunoimaging has been developed in recent years with increasing knowledge of molecular pathways of cancer cells. Based on a fundamental much alike targeted therapy, by modifying the tracer which target specific molecular of tumor cells, radioimmunoimaging helps provide tumor molecular information and thereby has a predictive value in response to targeted therapy and contributes to individualized medicine [62-64].

Since metabolic change in tumor always takes place earlier than anatomic change, PET has great advantage in early evaluation of treatment efficacy. Regarding to the widespread utilization of PET in assessment of therapy assessment, European Organization for Research and Treatment of Cancer (EORTC) and the National Cancer Institute (NCI) proposed guidelines in 1999 and 2006, respectively, intending to standardize the methodology of data acquisition and analysis of ${ }^{18} \mathrm{~F}$-FDG PET scans $[65,66]$. A prospective trial assessed the relationship between PET-evaluated response and clinical outcomes by evaluating on-treatment changes in ${ }^{18} \mathrm{~F}$-FDG and ${ }^{18} \mathrm{~F}$-FLT PET imaging among patients with NSCLC treated with targeted therapy and verified that it is possible to achieve high reproducibility of scan acquisition methodology, as long as strict imaging compliance guidelines are mandated in the study protocol [67]. Furthermore, the relevance for the prediction of clinical benefit of targeted therapy using different quantitative parameters for PET with both ${ }^{18} \mathrm{~F}$-FDG and ${ }^{18} \mathrm{~F}$-FLT in patients with advanced NSCLC, early ${ }^{18}$ F-FDG PET and ${ }^{18}$ F-FLT PET can predict PFS regardless of the method used for standardized uptake value (SUV) calculation although several indicators like $\mathrm{SUV}_{\max }$ measured with ${ }^{18} \mathrm{~F}-\mathrm{FDG}$ might be more robust to use for early response prediction [68]. All these efforts offer a promising prospect of robust application of this new modality in efficacy evaluation of tumors. With more targeted drugs acting on various mechanisms increasingly coming out, as well as diverse tumor metabolic characteristics, further researches are still required to offer a valid methodology for PET as a biomarker for early evaluation of targeted therapy.

While conventional imaging modalities are fraught with difficulties in early response assessment to cytostatic targeted therapy, ${ }^{18} \mathrm{~F}-\mathrm{FDG}$ PET meets these challenges both theoretically and practically. Although the reliability and powerfulness of ${ }^{18} \mathrm{~F}$-FDG PET in predicting therapeutic efficacy in NSCLC has been verified in chemotherapy in the last few years [69-72], there are fewer clinical studies on its efficiency in early prediction of outcome in targeted therapy except for gastrointestinal stromal tumor (GIST) [73-75]. However, preclinical studies have shown evidences that PET may be a promising biomarker for targeted therapies [7679].

2.6. Single-Photon Emission-Computed Tomography (SPECT). SPECT is also a molecular imaging technique, detecting tracer labeled with radionuclide emitting single gamma rays. The dual modality of SPECT/CT has been used in lung cancer diagnosis [80]. The most investigated trace used in treatment of lung cancer is ${ }^{99 \mathrm{~m}} \mathrm{Tc}$-methoxyisobutylisonitrile $\left({ }^{99 m} \mathrm{Tc}-\mathrm{MIBI}\right)$, which is reported to play a role in recognition of chemoresistance of tumor cell [81] and thus has been used in prediction of response to chemotherapy for NSCLC. A systematic review of the literature revealed that ${ }^{99 \mathrm{~m}} \mathrm{Tc}-\mathrm{MIBI}$ SPECT can accurately identify patients with lung cancer who will respond to chemotherapy, with an overall sensitivity of $94 \%$, specificity of $90 \%$, and accuracy of $92 \%$ [82]. In comparison with PET, SPECT has a lower sensitivity, and the use of SPECT in assessment of treatment of NSCLC is still limited.

2.7. Ultrasound. Ultrasound generates images by detecting changes of sound speed caused by various tissue densities. Since lung is filled with air which hardly echoes, ultrasound, together with a more recent utilization as endoscopic ultrasound (EUS), is not a usual technique used in NSCLC except for mediastinal staging or guiding fine needle aspiration or a metastasis lesion evaluation.

\section{Molecular Imaging in Response Evaluation of Targeted Therapy for NSCLC}

Molecular imaging modalities are now under evaluation in targeted therapy for advanced NSCLC although limitedly. In various agents targeting diverse pathways of cancer cell, there are only several agents targeting EGFR, and VEGFR pathways have been evaluated by molecular imaging, entirely PET in clinical or preclinical studies.

3.1. EGFR Pathway. EGFR (also known as HER [human EGF receptor] and ErbB1) is member of the human epidermal growth factor receptor family. By triggering the downstream signaling pathways involving phosphatidylinositol-3$\mathrm{OH}$ kinase $(\mathrm{PI}(3) \mathrm{K}) / \mathrm{AKT} / \mathrm{mammalian}$ target of rapamycin (mTOR) and RAS/RAF/MEK and signal transducer and activator of transcription (STAT) [83], the activation of EGFR contributes to tumor growth and progression, including proliferation, maturation, angiogenesis, invasion, metastasis, and inhibition of apoptosis [84]. Overexpression or aberrant activation of EGFR is frequent in NSCLC $[85,86]$, and inhibition of EGFR is a rational anticancer strategy, which have been validated in clinical practice. EGFR is a transmembrane glycoprotein, consisting of an extracellular ligand-binding domain, a transmembrane region, and an intracellular tyrosine kinase domain [87]. Two classes of agents targeting EGFR are used in clinical practice: tyrosine kinase inhibitors (TKIs) and monoclonal antibodies. 
3.1.1. EGFR TKIs. TKIs are small molecules that inhibit phosphorylation of EGFR intracellular tyrosine kinase by competing with ATP, leading to cell circle delay, inhibition of angiogenesis and apoptosis [88, 89]. In all the agents of EGFR TKIs being evaluated in clinical or preclinical studies, erlotinib is the only EGFR-targeting agent approved by FDA for the treatment of NSCLC. Gefitinib is another EGFR TKI that has been extensively investigated.

The value of ${ }^{18} \mathrm{~F}$-FDG PET in predicting efficacy of EGFR antagonist was validated in a preclinical study performed in 2006 [77]. A dramatic decrease in ${ }^{18}$ F-FDG uptake was observed at as early as 2 hours after treatment in gefitinibsensitive cell lines, while no measurable changes in ${ }^{18} \mathrm{~F}$-FDG uptake were seen in gefitinib-resistant cells.

A clinical study for early prediction of the efficacy of gefitinib for NSCLC conducted in 2007 monitored tumor response by both CT and ${ }^{18} \mathrm{~F}-\mathrm{FDG}$ PET [90]. In this study, two of the five NSCLC patients who exhibited stable disease according to RECIST criteria at 4 weeks after initiation of treatment but with long-term PFS (12.9 and 12.5 months) showed a marked decrease in ${ }^{18} \mathrm{~F}$-FDG uptake (measured by $S U V_{\max }$ ) within 2 days of treatment initiation, while $\mathrm{SUV}_{\text {max }}$ increase on day 2 had proved progressive disease based on CT evaluation. Although conclusions can be hardly drawn due to the small sample size, it did show the potential of ${ }^{18} \mathrm{~F}$-FDG PET in early response evaluation for targeted therapy.

Another study observed an increase of $\mathrm{SUV}_{\max }$ as measured with ${ }^{18} \mathrm{~F}$-FDG PET after discontinuation of treatment with erlotinib or gefitinib, due to progressive disease according to RECIST, and reintroduction of erlotinib or gefitinib again resulted in decreases in $\mathrm{SUV}_{\max }$ [91]. A subsequent introduction of everolimus, the mTOR inhibitor, led to further decrease in $S U V_{\max }$ and tumor size after 3 weeks of combination therapy in 5 of the 10 patients, suggesting that some tumor cells remain sensitive to EGFR blockage after PD occurs according to RECIST.

A prospective multicenter trial evaluated response of seventy-four patients with advanced NSCLC to erlotinib treatment with only PET/CT scans [62], further verified early ${ }^{18} \mathrm{~F}-\mathrm{FDG}$ PET response is associated with improved PFS and OS, even in the absence of subsequent RECST evaluation with CT.

${ }^{18}$ F-FLT PET was also applied to early detection of targeted therapy efficacy. A striking and reproducible decrease in ${ }^{18} \mathrm{~F}$-FLT uptake was exhibited in erlotinib-sensitive tumors after two days of treatment and translated into dramatic tumor shrinkage four days later [92]. Another phase II clinical trial assessed the use of ${ }^{18} \mathrm{~F}$-FDG PET and ${ }^{18} \mathrm{~F}$-FLT PET in NSCLC patients following erlotinib therapy [93]. This study enrolled thirty-four previously untreated patients, demonstrated an that early ${ }^{18} \mathrm{~F}-\mathrm{FDG}$ response (cutoff value: $30 \%$ reduction in the peak standardized uptake value) predicted significantly longer PFS, OS, and nonprogression after 6 weeks of therapy with erlotinib, while early ${ }^{18}$ F-FLT response was only associated with significantly longer PFS. In particular, this study demonstrated early ${ }^{18}$ F-FDG PET can identify those patients who could be benefited from erlotinib even without the knowledge of EGFR mutation.
3.1.2. Monoclonal Antibodies. EGFR-directed monoclonal antibodies exert an anticancer action by binding to the extracellular domain of the EGFR. Among all EGFR antibodies, cetuximab has been investigated extensively in treatment of NSCLC. A large phase III randomized trial shows that adding cetuximab to chemotherapy (cisplatin/vinorelbine) slightly increases overall survival (11.3 versus 10.1 months, $P=0.04$ ) [94]. Other monoclonal antibodies directing EGFR are still in investigation in clinical trials.

A phase II trial evaluating ${ }^{18} \mathrm{~F}-\mathrm{FDG}$ PET and CT as endpoints for assessing efficacy of cetuximab in combination with chemotherapy for advanced gastric or gastroesophageal junction adenocarcinoma showed that ${ }^{18}$ F-FDG PET could correctly differentiate responders from nonresponders, with a median TTP 16 months and 11 months, respectively [95]. No clinical studies have been performed regarding molecular imaging in treatment of NSCLC with EGFRtargeted monoclonal antibodies, but the use of ${ }^{18} \mathrm{~F}$-FLT PET has been investigated in a study of human lung cancer xenografts [96] demonstrated that $S \mathrm{VV}_{\max }$ in tumor tissue significantly decreased versus nontreated control on day 3 after treatment with cetuximab $(P<0.01)$, while no difference in $\mathrm{CT}$ image was visualized until day 8.

3.2. VEGFR Pathway. VEGFR pathway plays an important role in angiogenesis, a process of endothelial cell division and migration resulting in formation of new capillaries [97]. Angiogenesis is critical to tumor growth and metastasis, and enhanced VEGF and its receptors expression is found in a variety of tumors [98]; thus, blockage of this pathway is an attractive strategy for anticancer treatment. VEGFR is a transmembrane receptor tyrosine kinase specifically expressed at the surface of endothelial cell [99]. Various antiangiogenic agents have developed in the last few years, and VEGFR-directed monoclonal antibodies and VEGFR TKI are the two classes of VEGFR agents currently used in studies of NSCLC antiangiogenesis treatment. Use of DCE MRI imaging as well as PET has been evaluated in this therapy concerning its effects on tumor vasculature. Despite the limit use of DCE MRI in evaluation of antiangiogenesis in NSCLC, DCE MRI is frequently used in other tumors. As a review exhibited, anti-angiogenic therapies do not always result in reductions of blood flow in the short term, and DCE MRI kinetic response relationships are not universally strong across all tissue sites for all drugs [100]. In treatment for patients with cancer other than NSCLC, ${ }^{18} \mathrm{~F}-\mathrm{FDG}$ PET exhibited a promising prospect in monitoring antiangiogenesis effect of VEGFR-directed pathway [100]. In VEGFR TKI no study with molecular imaging is available at present.

Bevacizumab is a humanized monoclonal antibody directed at VEGF. It gained FDA approval based on an Eastern Cooperative Oncology Group (ECOG) conducted study [101], in which an increase in survival (12.3 versus 10.3 months) was observed in selected NSCLC patients when added Bevacizumab to standard chemotherapy. 
Forty-seven chemonaive patients with advanced NSCLC treated with bevacizumab and erlotinib underwent both ${ }^{18} \mathrm{~F}$ FDG PET and DCE MRI scan after 3 weeks of treatment [58], $>20 \%$ decrease in SUV as measured with ${ }^{18}$ F-FDG PET is well predicative for longer PFS (9.7 versus 2.8 months; $P=0.01$ ), while $>40 \%$ decrease in $K^{\text {trans }}$ (the endothelial transfer constant) as assessed by DCE MRI did not predict for longer PFS though most tumors have a decrease in $K^{\text {trans }}$ value after 3 weeks of treatment.

\section{Conclusions and Future Directions}

This paper reviews the currently available studies of molecular imaging used as early prediction of efficacy of targeted therapy for patients with NSCLC. For patients with advanced NSCLC, although the application of chemotherapy does prolong the OS comparing with supportive care alone [7], the prognosis of NSCLC remains poor, with a median survival of mere 8-10 months [11]. Nevertheless, it is validated that different combinations of chemotherapeutic drugs produce similar response rate and survival $[7,8]$ and the efficacy of chemotherapy for NSCLC is generally accepted as having reached a plateau. Targeted therapy, developing with the increasing knowledge of tumor molecular pathways, has an approach of blocking specific pathways of tumor cells instead of conventional cytotoxic effect. This new therapy for NSCLC has been investigated in many clinical trials and yielded significant improvement in RR and PFS. As a focus of current studies on therapy for NSCLC, increasing diverse targeted agents is now being investigated in clinical trials or preclinical studies. Meanwhile, the development of targeted agents highlights the need of a robust modality in efficacy assessment because the current prevailing anatomy-based criteria established on the basis of numerous previous studies on chemotherapy can no longer satisfy the requirement for early diagnosis.

Along with molecular metabolic mechanisms of tumor cells that increasingly come to light, rapid development of molecular imaging has taken place in recent years [102]. By directly visualizing and measuring the biological process in vivo [103], molecular imaging enables early assessment of response to anticancer treatment. Although molecular imaging modalities such as PET, SPECT, and MRS are involved in diagnosis of NSCLC, PET with different radiotracers is the primary modality that has been investigated in early predication of targeted therapy for NSCLC. Despite limited studies are available by now regarding the application of PET in this area, the outcome is encouraging, exhibiting utilization of PET in response evaluation, and outcome prediction is feasible and accurate. Obviously, more investigations are needed. More researches on molecular imaging in early prediction of targeted therapy efficacy should be done considering encouraging outcomes of the available studies and infinite prospect of diverse molecular imaging modalities.

\section{Conflict of Interests}

The authors declare that they have no conflict of interests.

\section{Acknowledgment}

This work is partly sponsored by Grants from the Zhejiang Provincial Natural Science Foundation of China (Z2110230), Health Bureau of Zhejiang Province (2010ZA075 and 2011ZDA013), National Science Foundation of China (NSFC) (no. 81101023, 81170306, and 81173468), and Ministry of Science and Technology of China (2011CB504400 and 2012BAI13B06).

\section{References}

[1] A. Jemal, F. Bray, M. M. Center, J. Ferlay, E. Ward, and D. Forman, "Global cancer statistics," Cancer Journal for Clinicians, vol. 61, no. 2, pp. 69-90, 2011.

[2] S. H. Landis, T. Murray, S. Bolden, and P. A. Wingo, "Cancer Statistics, 1999," Ca-A Cancer Journal for Clinicians, vol. 49, no. 1, pp. 8-31, 1999.

[3] National Cancer Institute, Surveillance, Epidemiology and End Results, http://seer.cancer.gov/.

[4] E. E. Vokes, J. D. Bitran, and N. J. Vogelzang, "Chemotherapy for non-small cell lung cancer; The continuing challenge," Chest, vol. 99, no. 6, pp. 1326-1328, 1991.

[5] C. M. Haskell, "Chemotherapy and survival of patients with non-small cell lung cancer; A contrary view," Chest, vol. 99, no. 6, pp. 1325-1326, 1991.

[6] G. Buccheri, "Chemotherapy and survival in non-small cell lung cancer; The old vexata questio," Chest, vol. 99, no. 6, pp. 1328-1329, 1991.

[7] S. Burdett, S. Burdett, R. Stephens et al., "Chemotherapy in addition to supportive care improves survival in advanced non-small-cell lung cancer: a systematic review and metaanalysis of individual patient data from 16 randomized controlled trials," Journal of Clinical Oncology, vol. 26, no. 28, pp. 4617-4625, 2008.

[8] F. Grossi, M. Aita, C. Defferrari et al., "Impact of thirdgeneration drugs on the activity of first-line chemotherapy in advanced non-small cell lung cancer: a meta-analytical approach," Oncologist, vol. 14, no. 5, pp. 497-510, 2009.

[9] J. H. Schiller, D. Harrington, C. P. Belani et al., "Comparison of four chemotherapy regimens for advanced non-small-cell lung cancer," New England Journal of Medicine, vol. 346, no. 2, pp. 92-98, 2002.

[10] F. A. Shepherd, "Chemotherapy for non-small cell lung cancer: have we reached a new plateau?" Seminars in Oncology, vol. 26, supplement 4, pp. 3-11, 1999.

[11] A. Martoni, A. Marino, F. Sperandi et al., "Multicentre randomised phase III study comparing the same dose and schedule of cisplatin plus the same schedule of vinorelbine or gemcitabine in advanced non-small cell lung cancer," European Journal of Cancer, vol. 41, no. 1, pp. 81-92, 2005.

[12] M. J. Duffy, "Predictive markers in breast and other cancers: a review," Clinical Chemistry, vol. 51, no. 3, pp. 494-503, 2005.

[13] M. J. Duffy and J. Crown, "A personalized approach to cancer treatment: how biomarkers can help," Clinical Chemistry, vol. 54, no. 11, pp. 1770-1779, 2008.

[14] H. Francis and B. Solomon, "The current status of targeted therapy for non-small cell lung cancer," Internal Medicine Journal, vol. 40, no. 9, pp. 611-618, 2010.

[15] T. S. Mok, Y. L. Wu, S. Thongprasert et al., "Gefitinib or carboplatin-paclitaxel in pulmonary adenocarcinoma," New England Journal of Medicine, vol. 361, no. 10, pp. 947-957, 2009. 
[16] D. H. Johnson, L. Fehrenbacher, W. F. Novotny et al., "Randomized phase II trial comparing bevacizumab plus carboplatin and paclitaxel with carboplatin and paclitaxel alone in previously untreated locally advanced or metastatic non-small-cell lung cancer," Journal of Clinical Oncology, vol. 22, no. 11, pp. 2184-2191, 2004.

[17] A. Sandler, R. Gray, M. C. Perry et al., "Paclitaxel-carboplatin alone or with bevacizumab for non-small-cell lung cancer," New England Journal of Medicine, vol. 355, no. 24, pp. 25422550, 2006.

[18] C. Gridelli, C. Butts, F. Ciardiello, R. Feld, C. Gallo, and F. Perrone, "An international, multicenter, randomized phase III study of first-line erlotinib followed by second-line cisplatin/gemcitabine versus first-line cisplatin/gemcitabine followed by second-line erlotinib in advanced non-small-cell lung cancer: treatment rationale and protocol dynamics of the TORCH trial," Clinical Lung Cancer, vol. 9, no. 4, pp. 235 238, 2008.

[19] M. Maemondo, A. Inoue, K. Kobayashi et al., "Gefitinib or chemotherapy for non-small-cell lung cancer with mutated EGFR," New England Journal of Medicine, vol. 362, no. 25, pp. 2380-2388, 2010.

[20] R. Govindan, "Summary of the proceedings from the 10th annual meeting of molecularly targeted therapy in nonsmall cell lung cancer," Journal of Thoracic Oncology, vol. 5, supplement 6, no. 12, p. S433, 2010.

[21] F. Janku, I. Garrido-Laguna, L. B. Petruzelka, D. J. Stewart, and R. Kurzrock, "Novel therapeutic targets in non-small cell lung cancer," Journal of Thoracic Oncology, vol. 6, no. 9, pp. 1601-1612, 2011.

[22] J. E. Larsen, T. Cascone, D. E. Gerber, J. V. Heymach, and J. D. Minna, "Targeted therapies for lung cancer: clinical experience and novel agents," Cancer Journal, vol. 17, no. 6, pp. 512-527, 2011.

[23] A. Chang, "Chemotherapy, chemoresistance and the changing treatment landscape for NSCLC," Lung Cancer, vol. 71, no. 1, pp. 3-10, 2011.

[24] A. Inoue, T. Suzuki, T. Fukuhara et al., "Prospective phase II study of gefitinib for chemotherapy-naïve patients with advanced non-small-cell lung cancer with epidermal growth factor receptor gene mutations," Journal of Clinical Oncology, vol. 24, no. 21, pp. 3340-3346, 2006.

[25] K. Yamazaki, I. Kinoshita, N. Sukoh et al., "A phase II trial of gefitinib as first-line therapy for advanced non-small cell lung cancer with epidermal growth factor receptor mutations," British Journal of Cancer, vol. 95, no. 8, pp. 998-1004, 2006.

[26] D. M. Jackman, B. Y. Yeap, N. I. Lindeman et al., "Phase II clinical trial of chemotherapy-naïve patients $>$ or $=70$ years of age treated with erlotinib for advanced non-small-cell lung cancer," Journal of Clinical Oncology, vol. 25, no. 7, pp. 760766, 2007.

[27] N. Sunaga, Y. Tomizawa, N. Yanagitani et al., "Phase II prospective study of the efficacy of gefitinib for the treatment of stage III/IV non-small cell lung cancer with EGFR mutations, irrespective of previous chemotherapy," Lung Cancer, vol. 56, no. 3, pp. 383-389, 2007.

[28] L. V. Sequist, D. W. Bell, T. J. Lynch, and D. A. Haber, "Molecular predictors of response to epidermal growth factor receptor antagonists in non-small-cell lung cancer," Journal of Clinical Oncology, vol. 25, no. 5, pp. 587-595, 2007.

[29] P. M. Fidias, S. R. Dakhil, A. P. Lyss et al., "Phase III study of immediate compared with delayed docetaxel after front-line therapy with gemcitabine plus carboplatin in advanced nonsmall-cell lung cancer," Journal of Clinical Oncology, vol. 27, no. 4, pp. 591-598, 2009.

[30] T. E. Stinchcombe and M. A. Socinski, "Treatment paradigms for advanced stage non-small cell lung cancer in the era of multiple lines of therapy," Journal of Thoracic Oncology, vol. 4, no. 2, pp. 243-250, 2009.

[31] G. V. Scagliotti, P. Parikh, J. Von Pawel et al., "Phase III study comparing cisplatin plus gemcitabine with cisplatin plus pemetrexed in chemotherapy-naive patients with advancedstage non-small-cell lung cancer," Journal of Clinical Oncology, vol. 26, no. 21, pp. 3543-3551, 2008.

[32] O. P. Joon, S. W. Kim, S. A. Jin et al., "Phase III trial of two versus four additional cycles in patients who are nonprogressive after two cycles of platinum-based chemotherapy in nonsmall-cell lung cancer," Journal of Clinical Oncology, vol. 25, no. 33, pp. 5233-5239, 2007.

[33] I. Vergote, G. J. S. Rustin, E. A. Eisenhauer et al., "Re: new guidelines to evaluate the response to treatment in solid tumors [ovarian cancer]," Journal of the National Cancer Institute, vol. 92, no. 18, pp. 1534-1535, 2000.

[34] World Health Organization, WHO Handbook for Reporting Results of Cancer Treatment, World Health Organization, Geneva, Switzerland, 1979.

[35] Food and Drug Administration, Reinventing the Regulation of Cancer Drugs: Accelerating Approval and Expanding Access, National Performance Review, Washington, DC, USA, 1996.

[36] A. B. Sandler, J. Nemunaitis, C. Denham et al., "Phase III trial of gemcitabine plus cisplatin versus cisplatin alone in patients with locally advanced or metastatic non-small-cell lung cancer," Journal of Clinical Oncology, vol. 18, no. 1, pp. 122-130, 2000.

[37] C. Sederholm, G. Hillerdal, K. Lamberg et al., "Phase III trial of gemcitabine plus carboplatin versus single-agent gemcitabine in the treatment of locally advanced or metastatic non-small-cell lung cancer: the Swedish lung cancer study group," Journal of Clinical Oncology, vol. 23, no. 33, pp. 83808388, 2005.

[38] C. M. R. Lima, N. A. Rizvi, C. Zhang et al., "Randomized phase II trial of gemcitabine plus irinotecan or docetaxel in stage IIIB or stage IV NSCLC," Annals of Oncology, vol. 15, no. 3, pp. 410-418, 2004.

[39] F. Grossi, F. de Marinis, V. Gebbia et al., "A randomized phase II trial of two sequential schedules of docetaxel and cisplatin followed by gemcitabine in patients with advanced non-small-cell lung cancer," Cancer Chemotherapy and Pharmacology, vol. 69, no. 2, pp. 369-375, 2012.

[40] K. R. Birchard, J. K. Hoang, J. E. Herndon Jr, and E. F. Patz Jr, "Early changes in tumor size in patients treated for advanced stage nonsmall cell lung cancer do not correlate with survival," Cancer, vol. 115, no. 3, pp. 581-586, 2009.

[41] M. Buyse and P. Piedbois, "On the relationship between response to treatment and survival time," Statistics in Medicine, vol. 15, no. 24, pp. 2797-2812, 1996.

[42] M. Buyse, P. Thirion, R. W. Carlson, T. Burzykowski, G. Molenberghs, and P. Piedbois, "Relation between tumour response to first-line chemotherapy and survival in advanced colorectal cancer: a meta-analysis," The Lancet, vol. 356, no. 9227, pp. 373-378, 2000.

[43] Administration FaD, Guidance for Industry Clinical Trial Endpoints for the Approval of Cancer Drugs and Biologics, Services UDoHaH, Washington, DC, USA, 2005.

[44] M. Buyse, T. Burzykowski, K. Carroll et al., "Progressionfree survival is a surrogate for survival in advanced colorectal 
cancer," Journal of Clinical Oncology, vol. 25, no. 33, pp. 5218 5224, 2007.

[45] P. N. Lara Jr, M. W. Redman, K. Kelly et al., "Disease control rate at 8 weeks predicts clinical benefit in advanced non-small-cell lung cancer: results from Southwest oncology group randomized trials," Journal of Clinical Oncology, vol. 26, no. 3, pp. 463-467, 2008.

[46] Q. Shi and D. J. Sargent, "Meta-analysis for the evaluation of surrogate endpoints in cancer clinical trials," International Journal of Clinical Oncology, vol. 14, no. 2, pp. 102-111, 2009.

[47] H. Choi, "Critical issues in response evaluation on computed tomography: lessons from the gastrointestinal stromal tumor model," Current Oncology Reports, vol. 7, no. 4, pp. 307-311, 2005.

[48] J. C. Yang, L. Haworth, R. M. Sherry et al., "A randomized trial of bevacizumab, an anti-vascular endothelial growth factor antibody, for metastatic renal cancer," New England Journal of Medicine, vol. 349, no. 5, pp. 427-434, 2003.

[49] M. Fukuoka, S. Yano, G. Giaccone et al., "Multi-institutional randomized phase II trial of gefitinib for previously treated patients with advanced non-small-cell lung cancer," Journal of Clinical Oncology, vol. 21, no. 12, pp. 2237-2246, 2003.

[50] M. G. Kris, R. B. Natale, R. S. Herbst et al., "Efficacy of gefitinib, an inhibitor of the epidermal growth factor receptor tyrosine kinase, in symptomatic patients with non-small cell lung cancer: a randomized trial," Journal of the American Medical Association, vol. 290, no. 16, pp. 2149-2158, 2003.

[51] R. Perez-Soler, "Phase II clinical trial data with the epidermal growth factor receptor tyrosine kinase inhibitor erlotinib (OSI-774) in non-small-cell lung cancer," Clinical Lung Cancer, vol. 6, no. 1, pp. S20-S23, 2004.

[52] J. Wang, N. Wu, M. D. Cham, and Y. Song, "Tumor response in patients with advanced non-small cell lung cancer: perfusion CT evaluation of chemotherapy and radiation therapy," American Journal of Roentgenology, vol. 193, no. 4, pp. 10901096, 2009.

[53] J. S. W. Lind, M. R. Meijerink, A. M. C. Dingemans et al., "Dynamic contrast-enhanced CT in patients treated with sorafenib and erlotinib for non-small cell lung cancer: a new method of monitoring treatment?" European Radiology, vol. 20, no. 12, pp. 2890-2898, 2010.

[54] H. Choi, C. Charnsangavej, S. C. Faria et al., "Correlation of computed tomography and positron emission tomography in patients with metastatic gastrointestinal stromal tumor treated at a single institution with imatinib mesylate: proposal of new computed tomography response criteria," Journal of Clinical Oncology, vol. 25, no. 13, pp. 1753-1759, 2007.

[55] A. D. Smith, S. N. Shah, B. I. Rini, M. L. Lieber, and E. M. Remer, "Morphology, Attenuation, Size, and Structure (MASS) criteria: assessing response and predicting clinical outcome in metastatic renal cell carcinoma on antiangiogenic targeted therapy," American Journal of Roentgenology, vol. 194, no. 6, pp. 1470-1478, 2010.

[56] P. S. Tofts, G. Brix, D. L. Buckley et al., "Estimating kinetic parameters from dynamic contrast-enhanced T1- weighted MRI of a diffusable tracer: standardized quantities and symbols," Journal of Magnetic Resonance Imaging, vol. 10, no. 3, pp. 223-232, 1999.

[57] I. Lee and K. Shogen, "Mechanisms of enhanced tumoricidal efficacy of multiple small dosages of ranpirnase, the novel cytotoxic ribonuclease, on lung cancer," Cancer Chemotherapy and Pharmacology, vol. 62, no. 2, pp. 337-346, 2008.

[58] A. M. Dingemans, A. J. de Langen, V. van den Boogaart et al., "First-line erlotinib and bevacizumab in patients with locally advanced and/or metastatic non-small-cell lung cancer: a phase II study including molecular imaging," Annals of Oncology, vol. 22, no. 3, pp. 559-566, 2011.

[59] P. F. Daly and J. S. Cohen, "Magnetic resonance spectroscopy of tumors and potential in vivo clinical applications: a review," Cancer Research, vol. 49, no. 4, pp. 770-779, 1989.

[60] H. Hanaoka, Y. Yoshioka, I. Ito, K. Niitu, and N. Yasuda, "In vitro characterization of lung cancers by the use of $1 \mathrm{H}$ nuclear magnetic resonance spectroscopy of tissue extracts and discriminant factor analysis," Magnetic Resonance in Medicine, vol. 29, no. 4, pp. 436-440, 1993.

[61] W. A. Weber, V. Petersen, B. Schmidt et al., "Positron emission tomography in non-small-cell lung cancer: prediction of response to chemotherapy by quantitative assessment of glucose use," Journal of Clinical Oncology, vol. 21, no. 14, pp. 2651-2657, 2003.

[62] L. Mileshkin, R. J. Hicks, B. G.M. Hughes et al., "Changes in ${ }^{18} \mathrm{~F}$-fluorodeoxyglucose and ${ }^{18} \mathrm{~F}$-fluorodeoxythymidine positron emission tomography imaging in patients with nonsmall cell lung cancer treated with erlotinib," Clinical Cancer Research, vol. 17, no. 10, pp. 3304-3315, 2011.

[63] A. Achmad, H. Hanaoka, H. Yoshioka et al., "Predicting cetuximab accumulation in KRAS wild-type and KRAS mutant colorectal cancer using (64) Cu-labeled cetuximab positron emission tomography," Cancer Science, vol. 103, no. 3, pp. 600-605, 2012.

[64] C. Wu, Z. Tang, W. Fan et al., "In vivo Positron Emission Tomography (PET) imaging of Mesenchymal - Epithelial Transition (MET) receptor," Journal of Medicinal Chemistry, vol. 53, no. 1, pp. 139-146, 2010.

[65] H. Young, R. Baum, U. Cremerius et al., "Measurement of clinical and subclinical tumour response using $\left[{ }^{18} \mathrm{~F}\right]-$ fluorodeoxyglucose and positron emission tomography: review and 1999 EORTC recommendations," European Journal of Cancer, vol. 35, no. 13, pp. 1773-1782, 1999.

[66] L. K. Shankar, J. M. Hoffman, S. Bacharach et al., "Consensus recommendations for the use of ${ }^{18} \mathrm{~F}$-FDG PET as an indicator of therapeutic response in patients in National Cancer Institute Trials," Journal of Nuclear Medicine, vol. 47, no. 6, pp. 1059-1066, 2006.

[67] D. S. Binns, A. Pirzkall, W. Yu et al., "Compliance with PET acquisition protocols for therapeutic monitoring of erlotinib therapy in an international trial for patients with non-small cell lung cancer," European Journal of Nuclear Medicine and Molecular Imaging, pp. 1-9, 2010.

[68] D. Kahraman, M. Scheffler, T. Zander et al., "Quantitative analysis of response to treatment with erlotinib in advanced non-small cell lung cancer using ${ }^{18} \mathrm{~F}-\mathrm{FDG}$ and 3'-deoxy-3'${ }^{18}$ F-fluorothymidine PET," Journal of Nuclear Medicine, vol. 52, no. 12, pp. 1871-1877, 2011.

[69] L. F. de Geus-Oei, H. F. M. Van Der Heijden, E. P. Visser et al., "Chemotherapy response evaluation with ${ }^{18} \mathrm{~F}-\mathrm{FDG}$ PET in patients with non-small cell lung cancer," Journal of Nuclear Medicine, vol. 48, no. 10, pp. 1592-1598, 2007.

[70] C. Nahmias, W. T. Hanna, L. M. Wahl, M. J. Long, K. F. Hubner, and D. W. Townsend, "Time course of early response to chemotherapy in non-small cell lung cancer patients with ${ }^{18}$ F-FDG PET/CT," Journal of Nuclear Medicine, vol. 48, no. 5, pp. 744-751, 2007.

[71] L. Decoster, D. Schallier, H. Everaert et al., "Complete metabolic tumour response, assessed by 18 -fluorodeoxyglucose positron emission tomography $\left({ }^{18} \mathrm{FDG}-\mathrm{PET}\right)$, after induction chemotherapy predicts a favourable outcome in patients with locally advanced non-small cell lung cancer (NSCLC)," Lung Cancer, vol. 62, no. 1, pp. 55-61, 2008. 
[72] Y. Imamura, K. Azuma, S. Kurata et al., "Prognostic value of SUVmax measurements obtained by FDG-PET in patients with non-small cell lung cancer receiving chemotherapy," Lung Cancer, vol. 71, no. 1, pp. 49-54, 2011.

[73] A. D. van den Abbeele and R. D. Badawi, "Use of positron emission tomography in oncology and its potential role to assess response to imatinib mesylate therapy in gastrointestinal stromal tumors (GISTs)," European Journal of Cancer, vol. 38, pp. S60-65, 2002.

[74] G. Antoch, J. Kanja, S. Bauer et al., "Comparison of PET, $\mathrm{CT}$, and dual-modality PET/CT imaging for monitoring of imatinib (STI571) therapy in patients with gastrointestinal stromal tumors," Journal of Nuclear Medicine, vol. 45, no. 3, pp. 357-365, 2004.

[75] S. Stroobants, J. Goeminne, M. Seegers et al., " ${ }^{18}$ FDGPositron emission tomography for the early prediction of response in advanced soft tissue sarcoma treated with imatinib mesylate (Glivec®)," European Journal of Cancer, vol. 39, no. 14, pp. 2012-2020, 2003.

[76] H. Prenen, C. Deroose, P. Vermaelen et al., "Establishment of a mouse gastrointestinal stromal tumour model and evaluation of response to imatinib by small animal positron emission tomography," Anticancer Research, vol. 26, no. 2, pp. 1247-1252, 2006.

[77] H. Su, C. Bodenstein, R. A. Dumont et al., "Monitoring tumor glucose utilization by positron emission tomography for the prediction of treatment response to epidermal growth factor receptor kinase inhibitors," Clinical Cancer Research, vol. 12, no. 19, pp. 5659-5667, 2006.

[78] S. Assadian, A. Aliaga, R. F. Del Maestro, A. C. Evans, and B. J. Bedell, "FDG-PET imaging for the evaluation of antiglioma agents in a rat model," Neuro-Oncology, vol. 10, no. 3, pp. 292-299, 2008.

[79] D. S. Dorow, C. Cullinane, N. Conus et al., "Multi-tracer small animal PET imaging of the tumour response to the novel pan-Erb-B inhibitor CI-1033," European Journal of Nuclear Medicine and Molecular Imaging, vol. 33, no. 4, pp. 441-452, 2006.

[80] O. Schillaci, "Single-Photon Emission Computed Tomography/Computed Tomography in Lung Cancer and Malignant Lymphoma," Seminars in Nuclear Medicine, vol. 36, no. 4, pp. 275-285, 2006.

[81] D. Piwnica-Worms, M. L. Chiu, M. Budding, J. F. Kronauge, R. A. Kramer, and J. M. Croop, "Functional imaging of multidrug-resistant P-glycoprotein with an organotechnetium complex," Cancer Research, vol. 53, no. 5, pp. $977-$ 984, 1993.

[82] H. K. Mohan and K. A. Miles, "Cost-effectiveness of 99mTcsestamibi in predicting response to chemotherapy in patients with lung cancer: systematic review and meta-analysis," Journal of Nuclear Medicine, vol. 50, no. 3, pp. 376-381, 2009.

[83] S. Talapatra and C. B. Thompson, "Growth factor signaling in cell survival: implications for cancer treatment," Journal of Pharmacology and Experimental Therapeutics, vol. 298, no. 3, pp. 873-878, 2001.

[84] J. R. Woodburn, "The epidermal growth factor receptor and its inhibition in cancer therapy," Pharmacology and Therapeutics, vol. 82, no. 2-3, pp. 241-250, 1999.

[85] V. Rusch, J. Baselga, C. Cordon-Cardo et al., "Differential expression of the epidermal growth factor receptor and its ligands in primary non-small cell lung cancers and adjacent benign lung," Cancer Research, vol. 53, no. 10, pp. 2379-2385, 1993.

[86] F. R. Hirsch, M. Varella-Garcia, P. A. Bunn et al., "Epidermal growth factor receptor in non-small-cell lung carcinomas: correlation between gene copy number and protein expression and impact on prognosis," Journal of Clinical Oncology, vol. 21, no. 20, pp. 3798-3807, 2003.

[87] A. Wells, "EGF receptor," International Journal of Biochemistry and Cell Biology, vol. 31, no. 6, pp. 637-643, 1999.

[88] D. Busse, R. S. Doughty, T. T. Ramsey et al., "Reversible G1 arrest induced by inhibition of the epidermal growth factor receptor tyrosine kinase requires up-regulation of p27(KIP1) independent of MAPK activity," Journal of Biological Chemistry, vol. 275, no. 10, pp. 6987-6995, 2000.

[89] A. M. Petit, J. Rak, M. C. Hung et al., "Neutralizing antibodies against epidermal growth factor and ErbB-2/neu receptor tyrosine kinases down-regulate vascular endothelial growth factor production by tumor cells in vitro and in vivo: angiogenic implications for signal transduction therapy of solid tumors," American Journal of Pathology, vol. 151, no. 6, pp. 1523-1530, 1997.

[90] N. Sunaga, N. Oriuchi, K. Kaira et al., "Usefulness of FDGPET for early prediction of the response to gefitinib in nonsmall cell lung cancer," Lung Cancer, vol. 59, no. 2, pp. 203210, 2008.

[91] G. J. Riely, M. G. Kris, B. Zhao et al., "Prospective assessment of discontinuation and reinitiation of erlotinib or gefitinib in patients with acquired resistance to erlotinib or gefitinib followed by the addition of everolimus," Clinical Cancer Research, vol. 13, no. 17, pp. 5150-5155, 2007.

[92] R. T. Ullrich, T. Zander, B. Neumaier et al., "Early detection of Erlotinib treatment response in NSCLC by $3^{\prime}$-deoxy$3^{\prime}$ - $\left[{ }^{18} \mathrm{~F}\right]$-fluoro-L-thymidine $\left(\left[{ }^{18} \mathrm{~F}\right] \mathrm{FLT}\right)$ positron emission tomography (PET)," PLoS ONE, vol. 3, no. 12, Article ID e3908, 2008.

[93] T. Zander, M. Scheffler, L. Nogova et al., "Early prediction of nonprogression in advanced non-small-cell lung cancer treated with erlotinib by using [(18)F]fluorodeoxyglucose and $[(18) F]$ fluorothymidine positron emission tomography," Journal of Clinical Oncology, vol. 29, no. 13, pp. 1701-1708, 2011.

[94] R. Pirker, J. R. Pereira, A. Szczesna et al., "Cetuximab plus chemotherapy in patients with advanced non-small-cell lung cancer (FLEX): an open-label randomised phase III trial," The Lancet, vol. 373, no. 9674, pp. 1525-1531, 2009.

[95] F. D. Fabio, C. Pinto, F. L. Rojas Llimpe et al., "The predictive value of ${ }^{18} \mathrm{~F}-\mathrm{FDG}-\mathrm{PET}$ early evaluation in patients with metastatic gastric adenocarcinoma treated with chemotherapy plus cetuximab," Gastric Cancer, vol. 10, no. 4, pp. 221227, 2007.

[96] S. Takeuchi, S. Zhao, Y. Kuge et al., " ${ }^{18}$ F-fluorothymidine PET/CT as an early predictor of tumor response to treatment with cetuximab in human lung cancer xenografts," Oncology Reports, vol. 26, no. 3, pp. 725-730, 2011.

[97] E. Cabebe and H. Wakelee, "Role of anti-angiogenesis agents in treating NSCLC: focus on bevacizumab and VEGFR tyrosine kinase inhibitors," Current Treatment Options in Oncology, vol. 8, no. 1, pp. 15-27, 2007.

[98] N. Ferrara, "The role of vascular endothelial growth factor in pathological angiogenesis," Breast Cancer Research and Treatment, vol. 36, no. 2, pp. 127-137, 1995.

[99] N. Weidner, J. P. Semple, W. R. Welch, and J. Folkman, "Tumor angiogenesis and metastasis - Correlation in invasive breast carcinoma," New England Journal of Medicine, vol. 324, no. 1, pp. 1-8, 1991.

[100] I. M. E. Desar, C. M. L. van Herpen, H. W. M. van Laarhoven, J. O. Barentsz, W. J. G. Oyen, and W. T. A. van der Graaf, 
"Beyond RECIST: molecular and functional imaging techniques for evaluation of response to targeted therapy," Cancer Treatment Reviews, vol. 35, no. 4, pp. 309-321, 2009.

[101] A. Sandler, R. Gray, M. C. Perry et al., "Paclitaxel-carboplatin alone or with bevacizumab for non-small-cell lung cancer," New England Journal of Medicine, vol. 355, no. 24, pp. 25422550, 2006.

[102] M. A. Pysz, S. S. Gambhir, and J. K. Willmann, "Molecular imaging: current status and emerging strategies," Clinical Radiology, vol. 65, no. 7, pp. 500-516, 2010.

[103] D. A. Mankoff, "A definition of molecular imaging," Journal of Nuclear Medicine, vol. 48, no. 6, pp. 18N-21N, 2007. 


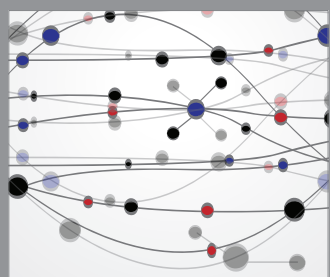

The Scientific World Journal
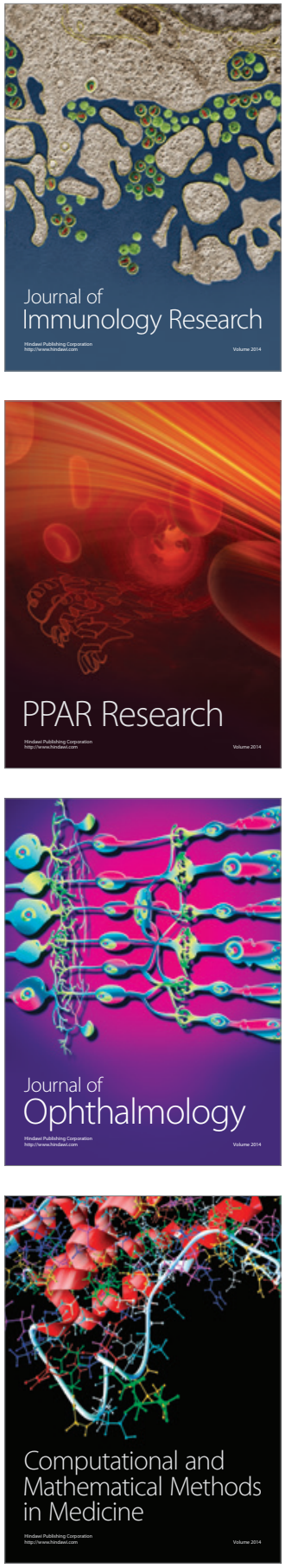

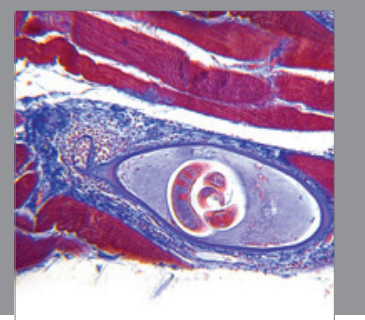

Gastroenterology

Research and Practice
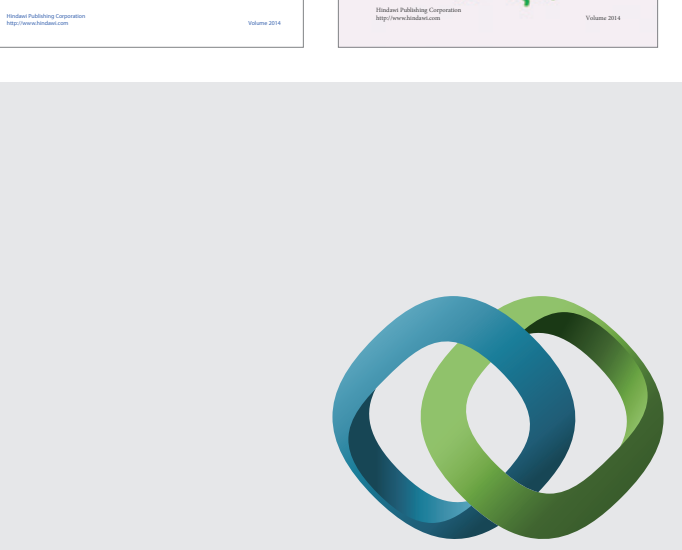

\section{Hindawi}

Submit your manuscripts at

http://www.hindawi.com
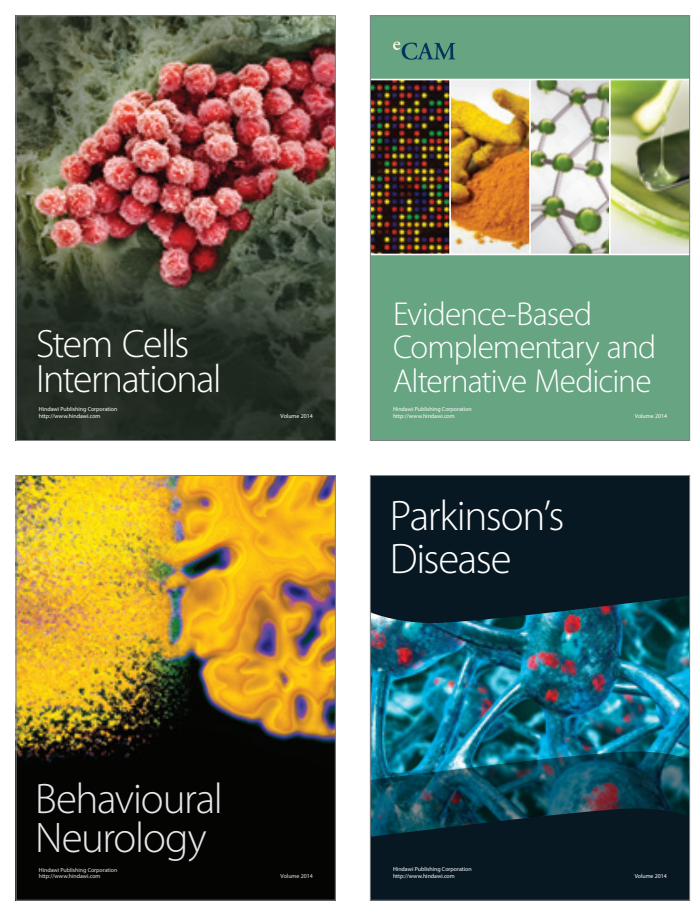

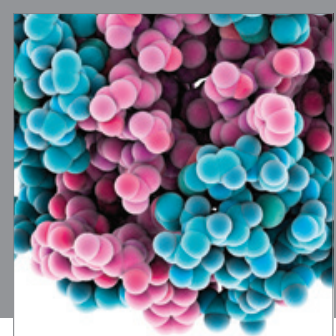

Journal of
Diabetes Research

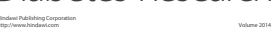

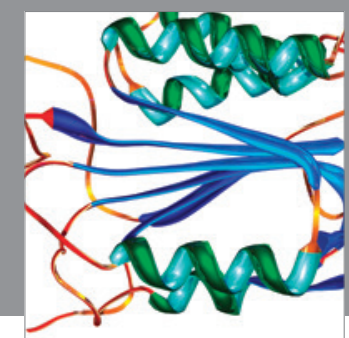

Disease Markers
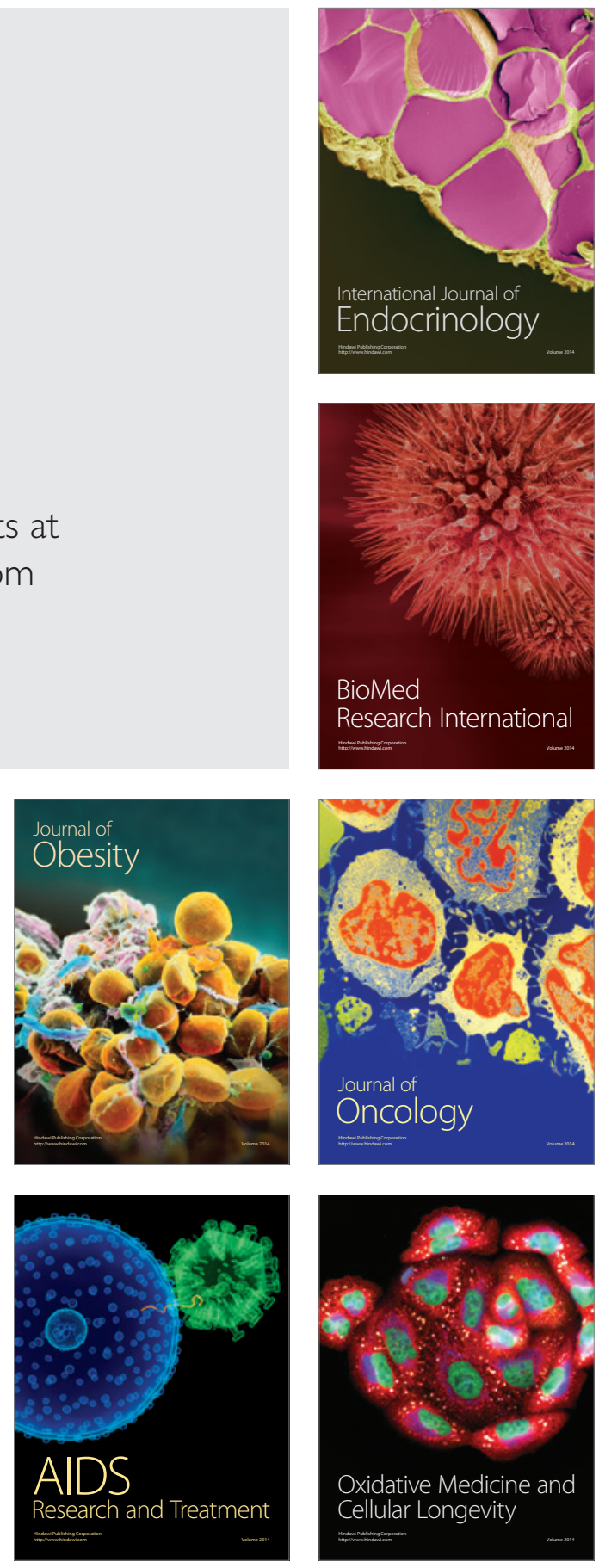